\section{El valor razonable en los estados financieros, un reto para las cooperativas'}

The fair value in the financial statements, a challenge for cooperatives

\author{
Orlando Quintero Bautista* \\ Óscar Ernesto Sánchez Martínez** \\ Mauricio Rodríguez Yanquen ${ }^{\star * *}$ \\ Heriberto Sánchez Montaña****
}

Recibido: 02-10-2014 / Revisado: 07-11-2014 / Aceptado: 09-04-2015

\section{Resumen}

En el país existen tres sectores de la economía de alto impacto en el PIB, como son el sector público, el sector privado y el sector de economía solidaria. Sobre el primer y segundo sector se encuentran trabajos muy bien elaborados sobre el significado de la aplicación del valor razonable, pero

1 Artículo de revisión.

* Economista de la Universidad Pedagógica y tecnológica de Colombia, contador público de la Universidad de Boyacá, especialista en Auditoría de Sistemas de la Universidad Antonio Nariño, docente investigador de la Universidad de Boyacá, Facultad de Ciencias Administrativas y Contables. oquintero@uniboyaca.edu.co

** Contador público de la Universidad de Boyacá, magíster en Administración Financiera y Económica, docente investigador de la Universidad de Boyacá y director del programa de contaduría pública, Facultad de Ciencias Administrativas. esanchezm@uniboyaca.edu.co

*** Contador público de la Universidad de Boyacá, magíster en Administración y Dirección de Empresas, docente investigador de la Universidad de Boyacá, Facultad de Ciencias Administrativas y contables. maurodriguez@uniboyaca.edu.co

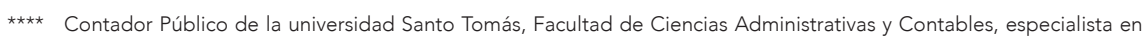
Revisoría Fiscal, candidato a magíster en Auditoría Internacional, docente investigador Universidad de Boyacá. hersanchez@ uniboyaca.edu.co sobre el tercer sector (cooperativas) poco se conoce a nivel nacional2.

Este articulo presenta un avance de la investigación que se viene desarrollando sobre El impacto del valor razonable en los indicadores financieros en el sector cooperativo de Boyacá, estudio de casos, y también describe la manera en que el valor razonable, al ser un estimativo de hechos económicos que afectan los estados financieros, puede generar variaciones importantes en los índices de solvencia de las cooperativas.

2 Comentario de los autores, ya que la mayoría de las referencias corresponden a estudios elaborados en España sobre el sector cooperativo español y europeo. 


\section{Citación}

Quintero, O., Sánchez, O., Rodríguez, M., Sánchez, H., (2015). El valor

razonable en los estados financieros, un reto para las cooperativas. Visión

empresarial, 1 (1). 50-68.

\section{Abstract}

Este artículo hace también una revisión de algunos trabajos relacionados, a fin de entender por qué las cooperativas merecen un análisis independiente de los demás sectores económicos. La investigación requiere apoyarse en algunos modelos teóricos de valoración de los hechos económicos y de estimación de los niveles de solvencia de las empresas como el de modelo Z de Altman, para medir los resultados de los estudios de casos que resulten de la investigación.

Palabras clave: cooperativas, valor razonable, instrumentos financieros, costo histórico, costo de reposición.
In Colombia there are three sectors of the economy of high impact on GDP, such as the public sector, the private sector and solidarity economy sector. On the first and second sector there are very well elaborated works, about the meaning of the application of the fair value, but little is known on the third sector (cooperatives) in the country.

This article presents an advance of the research that has been being performed about The impact of the fair value in the financial indicators in the cooperative sector of Boyacá, case study, and also describes how a fair value, when it is an estimate of economic facts that affect the financial statements, can generate important variations in the credit ratings of the cooperatives.

This paper makes a review of some related works in order to understand why cooperatives deserve an independent analysis of the other economic sectors. This research needs to be supported by some theoretical valuation models of economic facts and estimation of solvency levels of the companies such as the $Z$ model of Altman, to measure the results of the case studies resulting from the research.

Keywords: cooperatives, fair value, financial instruments, historical cost, replacement cost. 


\section{Introducción}

La contabilidad es el conjunto sistemático de actividades realizadas para informar y valorar los bienes económicos, a fin de analizar el resultado de las metas propuestas. Lo que se intenta, por tanto, es la organización o documentación racional del esfuerzo humano para satisfacer sus necesidades con un mínimo de esfuerzo relativo. Su objetivo es un máximo de información con un mínimo de error. Y el hecho económico sería la racionalización del gasto con un máximo de beneficio. Condición que obliga a la contabilidad y la economía a actuar en constante interacción. En el presente artículo se hace una revisión del marco teórico y normativo que define el valor razonable al aplicar las normas internacionales de información financiera en el sector cooperativo ${ }^{3}$.

El reto actual de la contabilidad de las cooperativas implica, por una parte, mantener los registros en forma cronológica, analizando las causas y efectos de los hechos económicos y, por la otra, reconocer las causas y efectos de la incertidumbre y del tiempo sobre los recursos y obligaciones de las cooperativas, debiendo actualizar los valores históricos o descontando los valores futuros para traerlos a valor presente, de manera que se puedan presentar sus estados financieros a valor razonable.

El concepto de valor razonable viene siendo utilizado por muchos países en sus registros contables, con el objeto de medir sus activos, pasivos, gastos y costos. Pero no todos los países pueden aplicar las mismas variables o conceptos de medición, ni tampoco todas las empresas o entes económicos, a pesar de que las normas internacionales solo diferencian en tamaño tres tipos de empresa, como son las de public accountability (contabilidad del sector público), pymes ${ }^{4}$ y microempresas.

En Colombia, a partir de la expedición del Decreto 1314 de 2009, mediante el cual se obliga a adoptar (convergencia) los estándares internacionales de información financiera, todos los sectores de la economía nacional deben revisar la forma de adopción y sus efectos económicos con la presentación de los estados financieros, pero más allá de la forma, lo más importante para el contador y todos los stakeholders o partes interesadas, es su interpretación financiera y sus efectos en los indicadores financieros.

\footnotetext{
4 Public accountability significa que cotiza en mercados de capital (financieros, de seguros, de valores). Emite públicamente instrumentos de deuda y patrimonio, es una entidad del Estado, que es una entidad de negocios, aplica las IFRS plenos. Si no tiene accountability pública pero publica estados financieros de propósito general, aplica las IFRS para pymes. Una entidad del Estado que no es una entidad de negocios (= entidad gubernamental)

aplica las IPSAS. Mantilla (2011, p. 37)
}

3 Impacto del valor razonable en los indicadores financieros en el sector cooperativo de Boyacá, estudio de casos. Trabajo de investigación en desarrollo realizado en la línea de investigación estudios sectoriales, del grupo de investigación GISEDE, de la Facultad de Ciencias Administrativas y Contables de la Universidad de Boyacá 
Las Normas Internacionales de Contabilidad y de Información Financiera establecen que tanto los recursos como las obligaciones de una entidad deben ser presentados, en los respectivos estados financieros, a su valor razonable. Y para ello se establecen, de acuerdo con su naturaleza y características, métodos de valuación así como el tratamiento de las diferencias que se produzcan entre el valor razonable determinado y el valor en libros (Gómez, 2005, p. 35-44).

Ante la necesidad de adoptar las normas internacionales de contabilidad, ya sea por imposición normativa o por tener que competir en el contexto internacional, surge para el sector cooperativo el reto de trasladar a los estados financieros esta realidad económica que necesita la cooperativa para ajustar sus proyecciones $y$, a su vez, para trasmitir la mayor confianza posible a los asociados y a los entes de control.

Actualmente los entes económicos colombianos están en proceso de implementación de las Normas Internacionales de Información Financiera, conocidas como NIIF por sus siglas en español, o IFRS en inglés.

A nivel internacional existen muchos escritos al respecto, debido a que dichas normas ya han sido implementadas en más de cien países. Sin embargo, para los propósitos de este artículo, lo primero que se debe mencionar son las propias normas que tratan sobre el valor razonable en la presentación de estados financieros: las
Normas Internacionales de Información Financiera: NIIF 1 adopción por primera vez de las NIIF, NIIF 2 pagos basados en acciones, NIIF 13 valor razonable; Normas Internacionales de Contabilidad: NIC 16 propiedades de inversión, NIC 18 ingresos ordinarios, NIC 22 costos por préstamos, NIC 32 instrumentos financieros: presentación, NIC 36 deterioro del valor de los activos, NIC 38 activos intangibles, NIC 39 instrumentos financieros: reconocimiento y medición, NIC 40 propiedad de inversión, y NIC 41 de agricultura; así como la SFAS 157 (Financial Accounting Standards, emitida por el FASB), entre las más sobresalientes, y la CINIIF 2, que hace referencia a las aportaciones de los socios en las cooperativas.

Con el objeto de organizar la presentación de las temáticas consultadas, se estructuró el presente artículo según los conceptos que componen el marco teórico del tema por resolver.

\section{Definición de cooperativismo}

La base para comprender por qué el sector solidario fue seleccionado para el presente estudio, debe extenderse al sentido mismo del concepto cooperativo, como función de solidaridad y trabajo en equipo, condición básica que rompe con el esquema cuantitativo de la presentación de los estados financieros y la participación de los socios en la distribución de las utilidades del negocio. Cambio que consiste en abandonar el sistema 
que otorga el poder de aplicar las decisiones a un grupo minoritario de personas, sustituyéndolo por un modelo de administración empresarial más democrático y participativo. Este criterio se revisó con la idea de soportar con estudios realizados la mejor forma de definir el modelo económico empresarial objeto de investigación.

La Comisión Científica del CIRIEC-España (citada por López \& Ochoa, 2002, p. 424), define la economía social, como

integrada por empresas privadas que actúan en el mercado produciendo bienes y servicios, asegurando o financiando la distribución del beneficio y toma de decisiones de tal forma que no estén directamente ligadas con el capital aportado por cada socio. El peso y la toma de decisiones es igual para todos los socios y nunca en función del capital aportado por cada uno de ellos. (Fernández, 2006).

Ladlaw (citado por Mateo, 1985) define la cooperativa como:

un grupo de personas, pequeño o grande, con el compromiso de asociar su acción sobre las bases de democracia y autoayuda en orden (sic) de asegurar un servicio o un acuerdo económico, que es a la vez, socialmente deseable y beneficioso para todos los que participan. (Mateo, 1985).
Jacques Defourny en su escrito titulado La economía social, entre economía capitalista y economía pública, manifiesta que "las cooperativas están, debido a sus normas particulares, en muy buena situación para constituir laboratorios de innovación social y de distribución del poder (Monzon \& Defourny, s.f.). Continúa en su mismo escrito, "es obvio que la economía social no posee todas las respuestas a esta cuestión, pero indica un camino transitable para una economía que esté más al servicio del hombre en su totalidad y de todos los hombres". (Monzon \& Defourny, s.f.).

La definición de cooperativa que propone la Alianza Cooperativa Internacional (citada por Zubiaurre, 2004) fija también un punto de partida para el presente trabajo, la cual define a las cooperativas como una

asociación autónoma de personas que se han unido de forma voluntaria para satisfacer sus necesidades y aspiraciones económicas, sociales y culturales en común mediante una empresa de propiedad conjunta y gestión democrática. (Zubiaurre, 2004).

A través de las cooperativas se pueden crear nuevas formas de gestión y generar un mayor aporte investigativo a los modelos de valoración de los componentes de los estados financieros, que permitan acercarse a la realidad financiera de la empresa sin la injerencia o presión de un dueño con el poder de decidir y con interés en obstaculizar la transparencia de los informes. 


\section{Modelo contable en las cooperativas}

Las empresas cooperativas no cuentan con una nueva normativa en la que se regulen, específicamente, los aspectos contables de estas. La ley 1314 de 2009 aprueba la adopción de las normas sobre los aspectos contables de las sociedades en general, las cuales tienen por objeto la adaptación de las normas de valoración y elaboración de cuentas anuales a las empresas que coticen en el mercado de valores, para las pymes, y las empresas simplificadas. Para el sector cooperativo, la Supersolidaria confirma en la circular externa 001 de 2013, los criterios mínimos a seguir en la presentación de información financiera, ratificando la clasificación de los grupos uno y tres para el sector solidario. De la misma forma, en la carta circular 001 del 2013, confirma los mismos criterios para el grupo dos, correspondientes al desarrollo de convergencia a los estándares internacionales, definidos en los decretos 2706 del 27 de diciembre de 2012 y 2784 de 28 de diciembre de 2012, donde se clasifican los grupos para la aplicación del marco técnico normativo. Quedando así, en nuestra opinión, huérfano el sector cooperativo de una ley que regule con claridad la aplicación contable para algunas cuentas especiales que son la esencia en la economía solidaria.

Magazín Empresarial muestra los resultados de una investigación sobre la economía solidaria y sus obstáculos normativos desde un enfoque económico.
Orjuela y Portocarrero en La economía solidaria en Colombia: obstáculos normativos y su enfoque económico, identifican y evalúan veintitrés problemas, con base en la aplicación del instrumento de análisis estructural en prospectiva Mic-Mac (matriz de impactos cruzados/multiplicación aplicada a una clasificación). Estos autores encontraron que el

sector solidario no tiene participación en entes u organismos consultivos estatales o gubernamentales de corte social, presenta limitaciones en el desarrollo normativo constitucional, restricciones originadas en la ley de creación del Dan social, la no existencia de unos adecuados sistemas de evaluación de las cooperativas según su actividad económica y ausencia de instituciones de fomento. (Orejuela \& Portocarrero, 2012).

Estos estudios siembran en el grupo investigador el interés por demostrar que es importante proponer al Gobierno nacional que regule la aplicación de normas contables en el sector de economía solidaria, que ayuden a entender sus estados financieros desde la perspectiva social y no meramente financiera y capitalista, como lo presentan las NIIF y NIC que se deben adoptar.

Un ejemplo de lo anterior es presentado por Miguel Ángel Zubiaurre en la revista Sociedades cooperativas aspectos contables singulares. El autor expresa lo siguiente:

ISSN 2462-9898 / Págs: 50 - 69 
es necesario volver a recordar que el capital pierde en las cooperativas dos de sus principales características en las sociedades mercantiles, es decir, no sirve para estructurar el derecho a voto ni tampoco como base para repartir beneficios o imputar pérdidas. (Zubiaurre, 2004).

\section{Impacto económico de las cooperativas en Boyacá}

Con el objeto de demostrar la importancia del trabajo, analizamos estudios realizados en el 2009 sobre el impacto de las cooperativas en la economía regional. Estos estudios indican que el empleo generado por el sector cooperativo en Boyacá tiene una participación importante dentro del total departamental y nacional. Encontramos un estudio hecho por Sonia Janeth Limas y Jairo Alberto Higuera, en el cual se analiza el impacto socioeconómico del sector de la economía solidaria en Boyacá 2000-2009, utilizando el método descriptivo. Los resultados muestran que este sector avanza hacia la consecución de un mayor posicionamiento en la economía solidaria del nivel nacional y departamental. Las cifras reflejan la dinámica socioeconómica del sector, su evolución y crecimiento. Los hallazgos anuncian nuevos estudios sobre el sector de la economía solidaria en Boyacá (Limas \& Higuera, 2012).

En Boyacá, el número de empleos generados por los fondos y las cooperativas durante el periodo 2000 -
2009 ascendió a 11.435 empleos, de los cuales el $97.58 \%$ correspondió a las cooperativas y el 2,47 \% a los fondos (Limas \& Higuera, 2012).

El Departamento de Análisis Económico de Confecoop presenta algunos elementos teóricos y prácticos que pretenden ilustrar sobre los movimientos económicos, las acciones emprendidas y los efectos que, en un marco macroeconómico, tienen las decisiones actuales de política económica para las empresas cooperativas. Igualmente, permiten comprender la dinámica económica reciente, con el fin de obtener mejores elementos de juicio para la toma de decisiones (Confecoop, 2008).

\section{Impacto de las NIIF en el sector cooperativo}

Al revisar el estado de la cuestión sobre los efectos de la aplicación de las normas internacionales en la contabilidad del sector cooperativo en Colombia, son muy pocos los documentos que podemos citar. Sin embargo, un trabajo de la Universidad Javeriana, realizado por Salazar-Baquero (2011), sobre las implicaciones de las NIIF en las pymes, determina los principales efectos no financieros en las medianas empresas en Colombia por la utilización de la NIIF para pymes en la presentación de reportes financieros. Para esto, analiza cada una de las secciones de la NIIF, organizadas por bloques temáticos, en busca de determinar las ventajas, problemáticas y demás implicaciones que puede repre- 
sentar el uso del estándar. El artículo explora las interrelaciones existentes con la contabilidad tributaria, las normas sobre derecho societario, y además establece los principales cambios en las prácticas contables que se esperan por la adopción de la NIIF para pymes. Sobresale el mayor uso de mediciones financieras (valor razonable, valor presente). También se precisan importantes diferencias con los requerimientos de la contabilidad tributaria, por lo que se espera que se incrementen las conciliaciones contables y tributarias y que haya pronunciamientos o modificaciones a las normas tributarias (Salazar-Baquero, 2011).

Un documento que aportó a la presente investigación sobre la necesidad de un modelo jurídico que regule el efecto contable en las cooperativas es el de Manuel Paniagua Zurera ${ }^{5}$ quien manifiesta en uno de sus apartes:

No obstante, las sociedades cooperativas no pueden quedar al margen de las Normas internacionales de contabilidad. La propia NIC 32 aporta el instrumento apropiado para calificar las aportaciones sociales cooperativas: los instrumentos financieros compuestos. Pero, el estudio de la inminente reforma del capital social cooperativo en la Ley estatal de cooperativas ponen de manifiesto precipitación y una recepción, acrí-

5 Profesor titular responsable de área de Derecho Mercantil, Facultad de Ciencias Económicas y Empresariales (ETEA). Institución Universitaria de la Compañía de Jesús, adscrita a la Universidad de Córdoba. tica, de las referidas Normas internacionales de contabilidad. (Paniagua, 2006).

Fernando Polo Garrido afirma en uno de sus artículos:

En estos momentos estamos viviendo un proceso de reforma contable, por este motivo se estudian los diferentes impactos que puede tener la aplicación de las Normas Internacionales de Información Financiera a las cooperativas. Finalmente se estudian los movimientos de respuesta surgidos a raíz de la CINIIF 2 más allá de nuestras fronteras. (Polo, 2006).

Josefina Fernández Guadaño hace un estudio sobre el criterio jurídico en el ámbito europeo, nacional y autonómico; el criterio contable nacional e internacional; y el criterio económico-financiero, del capital social de las cooperativas como resultado de la aplicación de las NIIF (Fernández- Guadaño, 2006).

Esta interpretación se aplicará a los instrumentos financieros que están dentro del alcance de la NIC 32, entre los que se incluyen los instrumentos financieros emitidos a favor de los socios de entidades cooperativas, que constituyen participaciones en la propiedad de dichas entidades. Esta Interpretación no será de aplicación a los instrumentos financieros que vayan a ser o puedan ser liquidados con instrumentos de patrimonio de la entidad (CINIIF, 2004). 
Uno de los temas que más se ha tratado con respecto al impacto de las NIIF en las cooperativas es el tratamiento contable de los aportes sociales en las cooperativas. En un trabajo realizado por la Universidad del Valle sobre el impacto de las NIIF en el sector cooperativo, una de sus conclusiones demuestra que se debe reglamentar mejor la composición del capital social y los fondos que constituyan el patrimonio de las cooperativas. Dicen en su artículo los investigadores:

La legislación cooperativa en Colombia debe dictar los lineamientos para el fortalecimiento del capital institucional de las cooperativas de ahorro y crédito, toda vez que este demuestra la seguridad y solidez de estas organizaciones, puesto que permite enjugar pérdidas y es fuente de financiación propia con costo cero, para apalancar proyectos en el mediano y largo plazo. (Rendón, Rodríguez \& Riascos, 2013).

\section{Aplicación de las NIIF en el sector cooperativo}

Teniendo en cuenta que se conocen pocos trabajos relacionados con la aplicación de las NIIF en el sector cooperativo en Colombia, debido a que la normatividad sobre la aplicación de las NIIF en el país tiene vigencia a partir de 2015 y que solo se sabrán los resultados de su aplicación en diciembre de 2016, se hará entonces referencia a algunos trabajos realizados sobre el tema en España:
Existe un trabajo de Fernando Polo Garrido (2007) que pretende identificar las áreas más relevantes (además de la clasificación de las aportaciones al capital social como patrimonio o pasivo financiero) en el régimen económico de las cooperativas que pueden verse afectadas por las Normas Internacionales de Información Financiera, en la medida en que sus criterios se vayan introduciendo en nuestro ordenamiento mercantil contable. Es decir, más allá de los supuestos obligatorios de aplicación de las NIIF (cuentas consolidadas de empresas cotizadas), con la finalidad de que sirva de base para futuras reformas legislativas cooperativas, a fin de mejorar su régimen económico, por una parte, y por otra, para adecuarlo a la mencionada nueva normativa mercantil-contable derivada de la adaptación a la actual normativa contable europea que adopta las NIIF. A este respecto hay que tener presente el mandato constitucional de fomento de las cooperativas mediante una legislación adecuada (Polo, 2007).

Salvador Sánchez hace un análisis de la necesidad de implementar un modelo contable especial a las cooperativas, debido a que estas presentan una serie de particularidades que las diferencian de las demás sociedades (Sánchez, 2003).

Miguel Ángel Zubiaurre Artola hace algunas apreciaciones sobre la definición y las características de las sociedades cooperativas, explica las principales particularidades contables de estas, los efectos en la 
determinación del resultado del ejercicio y el excedente de la cooperativa, a la luz de las normas internacionales de información financiera (Zubiaurre, 2004).

En el seminario de sensibilización sobre la aplicación de las NIIF en el sector cooperativo, presentado por Confecoop, se da una clara explicación de los efectos en la aplicación de dichos estándares de contabilidad y de la necesidad de regular el manejo del capital social de las cooperativas mediante una ley (Confecoop, 2012a).

\section{El valor razonable en las cooperativas}

Los señores Isaac Jonás González y Jorge Herrerosº, en su artículo Valor razonable y libro blanco, analizan la modificación de las directivas cuarta y séptima de la Unión Europea, así como la decisión de acercar la norma contable de los países europeos entre sí con el horizonte final de las normas internacionales de contabilidad, que ha puesto sobre la mesa la posibilidad de contabilizar determinados activos a su valor razonable. El artículo citado analiza las diferentes NIC en las que se contempla el valor razonable, prestando especial atención a la NIC 39 sobre instrumentos financieros, así como al tratamiento que debe darse a las diferentes

\footnotetext{
6 Economista y auditor censor jurado de cuentas. Exsocio de las firmas Arthur Andersen y Deloitte. Ha sido miembro permanente y ponente de la Comisión de Expertos para la Reforma Contable. Es vocal titular del Comité de Auditoria del Instituto de Contabilidad y Auditoría de Cuentas (ICAC). Jorge Herreros es socio del Departamento de Práctica Profesional de Auditoría de KPMG en España (página web aeca.es, s.f.).
}

operaciones de cobertura (González \& Herreros, 2002). EI IASB (International Accounting Standards Board) junto con el FASB (Financial Accounting Standards Board) presentaron el día 12 de mayo del año 2011 una guía para la medición a valor razonable, así como los requisitos de revelación para las NIIF y los US-GAAP (NICNIIF, 2011).

La guía expuesta en la NIIF 13 y en el asunto 820 en las Normas FASB, perfecciona el proyecto vinculado de ambas para optimizar las NIIF y los PCGA de los Estados Unidos y para conseguir su convergencia. Vale anotar que ese proyecto constituyó el trabajo conjunto de más de cinco años.

Para las NIIF, la NIIF 13 Medición a valor razonable mejorará la coherencia y reduce la complejidad al proporcionar, una definición precisa del valor razonable y los requisitos de revelación para el uso a través de las NIIF.

Para los US-GAAP, la actualización sustituye a la mayor parte de la orientación existente, aunque muchos de los cambios son aclaraciones de la orientación existente o cambios de redacción para alinearla con la NIIF 13. También refleja la consideración de FASB de las diferentes características de las entidades públicas y no públicas y las necesidades de los usuarios de sus estados financieros. Las entidades no públicas estarán exentas de una serie de nuevos requisitos de revelación. (NICNIIF, 2011). 
Fernando Polo Garrido (2007, p. 83-108) en su artículo hace referencia a la importancia del nuevo ordenamiento contable como base para que las futuras reformas legales que tengan injerencia en el sector cooperativo, contemplen todas las mejoras necesarias para apoyar el sector cooperativo en su verdadera razón de ser, como es el desarrollo social justo y equitativo de la economía solidaria.

Sánchez Jiménez (2003), quien analiza los principales factores que determinan la necesidad de una regulación contable específica para el sector cooperativo, resalta las diferencias con los demás sectores de la economía en cuanto a su interés mercantil, su relación cliente-proveedor, trabajadores y dueños, y la denominación de excedente empresarial, por lo que no puede ser generalizado por los estándares internacionales de contabilidad.

En resumen, el informe plantea que el tratamiento de las operaciones que realizan las cooperativas con sus socios debe hacerse contablemente de acuerdo con su verdadero valor económico y según el criterio de valor razonable o fair value.

María del Carmen López Pérez (2005) se sustenta básicamente en la teoría del valor expuesta por las escuelas clásicas, la cual indica que los fisiócratas tocan el concepto del valor. Además, López (2005) cita a Petty, Loke y Cantillon, quienes contemplaron la relación del valor con el trabajo. Igualmente menciona a Adam
Smith, con el objeto de clarificar los conceptos de valor de uso y valor de cambio, nociones que aportan al trabajo bases para soportar el marco teórico del tema por tratar.

El trabajo de Carmen López (2005) resalta como a través del estudio de los clásicos de la economía, se pueden encontrar bases sólidas que expliquen el verdadero valor económico de las cosas y su precio natural.

Slof, Rodríguez, Torvent, Villadell y Sola (2005) escribieron acerca de la cuantificación del efecto sobre los indicadores financieros del cambio del costo histórico a valor razonable, en la valoración de los activos financieros y el inmovilizado material.

El trabajo de María del Carmen Benavente Martínez y María Eva Vallejo Pascual (2002), puede ser importante para este estudio en la medida en que se haga una revisión de las cooperativas del sector agrícola en Boyacá. Con esta revisión se pretende conocer la situación actual de utilización del valor razonable por las grandes empresas, valorando la aceptación de dicho criterio y el impacto que su utilización está teniendo no solo en los resultados empresariales, sino en los principales indicadores de la situación económico-financiera de las empresas agrícolas.

Zúñiga, Pacheco y Díaz (2009, p. 75) publicaron un artículo que se apoya principalmente en la revisión 
de la bibliografía relacionada con la convergencia del proceso de internacionalización de las empresas chilenas. Este trabajo despertó nuestro interés, debido a las conclusiones presentadas, entre ellas, la afectación de ciertas cuentas contables, por la aplicación del valor razonable en cuentas de resultados, patrimonio y activos fijos, de tal forma que los ratios financieros presentan diferencias en su comparabilidad y, a su vez, afectan las decisiones de los administradores de las empresas, como resultado de los cambios económicos significativos evidenciados con la presentación de los estados financieros.

\section{Modelos Z de Altman}

Con el objeto de ajustar el marco teórico de este modelo a la precisión de sus planteamientos, se transcribe la definición expresada por el ingeniero Alejandro Atorga Hilbert ${ }^{7}$, secretario técnico del IFECOM ${ }^{8}$ :

\section{El Modelo Z fue creado por Edward Altman' con base en un análisis estadístico interactivo de discriminación múltiple en el que se ponderan y suman cinco razones de medición para clasificar las empresas en solventes e insolventes.}

Para el desarrollo de su modelo en 1966, Altman tomó una muestra de 66 empresas, de las cuales 33 habían quebrado durante los 20 años anteriores y 33 seguían operando a esa fecha. A la muestra le calculó 22 razones financieras que clasificó en 5 categorías estándar: liquidez, rentabilidad, apalancamiento, solvencia y actividad. Después de numerosas corridas, se seleccionaron las 5 variables que, juntas, dieron el mejor resultado en la predicción de la insolvencia. Esto se hizo mediante: 1. Observación estadística de varias funciones, incluyendo la contribución relativa de cada variable independiente. 2. Evaluación de intercorrelaciones entre las variables relevantes. 3. Observación de la precisión en la predicción de los modelos y, 4. Criterio del analista.

\footnotetext{
9 El profesor Altman recibió su MBA y Ph.D. en Finanzas en la Universidad de California. Fue ingresado dentro del Salón de la Fama del "Fixed Income Analysts Society" en 2001, fue nombrado presidente de la Financial Management Association (2003). En 2005, el profesor Altman fue nombrado como una de las "100 personas más influyentes en finanzas" por la revista Treasury \& Risk Management. Es editor ejecutivo de la publicación internacional Journal of Banking and Finance y editor consultivo de la serie John Wiley Frontiers in Finance Series. Ha publicado 20 libros y alrededor de 150 artículos en revistas académicas de finanzas, contabilidad y economía. Fue el editor del Handbook of Corporate Finance y del Handbook of Financial Markets and Institutions, y es autor de los libros: Bankruptcy, Credit Risk and High Yield Junk Bonds (2002), Recovery Risk (2005), Corporate Financial Distress \& Bankruptcy (2006) y Managing Credit Risk (2008), (citados en página web de riskmathics.com).
}

\footnotetext{
7 Experiencia en la banca de más de doce años, con puestos en diferentes niveles y áreas, en tres instituciones bancarias, desde analista financiero, ejecutivo de cuenta, gerente de crédit en Banamex, subdirector de banca corporativa en Ixe Banco, subdirector Banca Empresaria Región Bajío Inverlat, hasta director de la Banca Empresarial de la División Metropolitan
}

8 INFECON: Instituto Federal de Especialistas de Concursos Mercantiles. 


\section{La función discriminante final fue:}

$Z=1.2 \times 1+1.4 \times 2+3.3 \times 3+0.6 \times 4+0.99 \times 5$

Donde: $X 1$ = capital de trabajo / activo total; $X 2=$ utilidades retenidas / activo total; $X 3=$ utilidades antes de intereses e impuestos / activo total; $\times 4=$ valor de mercado del capital / pasivo total $\mathrm{y}_{;} \times 5=$ ventas / activo total.

El resultado indica que, si $Z>=2.99$, la empresa no tendrá problemas de insolvencia en el futuro; si $Z<=1.81$, entonces es una empresa que de seguir así, en el futuro tendrá altas posibilidades de caer en insolvencia. El modelo considera que las empresas se encuentran en una "zona gris" o no bien definida, si el resultado de Z se encuentra entre 1.82 y 2.98 .

Debido a que este modelo aplicaba solo a empresas manufactureras que cotizaban en bolsa, Altman hizo una revisión del mismo y obtuvo dos nuevas versiones, el Z1 y el Z2.

\section{Expectativas del proyecto}

Tomando las entidades del sector cooperativo de Boyacá, como las que más impulsan el desarrollo y crecimiento económico de la región ${ }^{10}$, es importante

10 En el año 2000, el PIB del sector representó el 1.79 \% del PIB nacional. Su participación aumentó en el 2002 a $4.42 \%$; a partir del 2005 se incrementó la participación, hasta alcanzar el $5.36 \%$ en el 2006. Desde el 2007 se presenta un decrecimiento hasta llegar al $4.96 \%$ en el 2009 (Limas \& Higuera, 2012). establecer por medio de esta investigación los efectos en los resultados financieros y sus indicadores del periodo contable, al aplicar la medición a valor razonable a sus estados financieros.

La evolución de las normas contables a nivel internacional han venido siendo impactadas por las exigencias del mercado, uno de estos impactos ha sido la evolución hacia el uso de los valores justos o razonables como elemento de medición de los estados contables, en algunos casos sustituyendo y en otros complementando la valuación al costo histórico que se viene aplicando según los PCGA.

Puesto que a los asociados les interesa saber cuál será el efecto tanto en su participación patrimonial como en los beneficios obtenidos a través de los resultados operacionales, se requiere revelar un análisis del impacto que este tipo de valoración tiene sobre la viabilidad económica de la entidad cooperativa, para que los asociados puedan tomar decisiones sobre el futuro de su participación.

La aplicación del método del valor razonable en nuestro país debe ser considerado en el nuevo plan contable que deben aplicar las cooperativas a partir de la implementación de las NIIF. Este principio reemplazaría en parte al principio de prudencia y valuación al costo o mercado, el que resulte menor. El valor razonable entonces se constituye en un reto para los 
contadores en ejercicio y para académicos, gerentes, inversionistas y legítimos interesados en los estados financieros, sirviendo de base para la toma de decisiones en materia financiera y de negocios en general. 


\section{Referencias}

Astorga, A. (s.f.). Estudio. Recuperado de www.ifecom.cjf.gob.mx/PDF\%5Cestudio\%5C3.pdf

Benavente, M.C. \& Vallejo, M.E. (año). Estudio empírico del impacto del valor razonable en la contabilidad de las empresas agrarias. León: Universidad de León, Área de Estadística e Investigación Operativa, Facultad de Ciencias Económicas y Empresarial.

CINIIF. (2004, noviembre). Aportaciones de socios de entidades cooperativas e instrumentos similares. CINIIF 2.

Confecoop. (2008). Actualidad económica e incidencia en el sector cooperativo. Observatorio Cooperativo, (4), 2-12.

Confecoop. (2012a). Proceso de sensibilización aplicación NIIF en cooperativas. Cali: Confecoop.

Confecoop. (2012b). Sector cooperativo colombiano primer semestre de 2012. Observatorio Cooperativo, (30), 2-17.

Fernández-Guadaño, J. (2006). Diferentes consideraciones en torno al capital social de las sociedades cooperativas. Revesco, (88), 40-61.

Fernández S., M. E. (2006). Las cooperativas: organizaciones de la economía. Revista de Ciencias Sociales RCS, 237-253. 
Gómez, M. (2005). Evaluación del enfoque de las NIIF desde la teoría de la contabilidad y el control. Revista Internacional Legis de Contabilidad \& Auditoría, (22), 149-195.

González, I.J. \& Herreros, J. (2002). Valor razonable y libro blanco. Partida Doble, 36, 78-89.

International Accounting Standards Board -IASB-. (2010). CNIFF 2. Aportaciones de socios de entidades cooperativas e instrumentos similares. Londres, UK: IASB.

International Accounting Standards Board (IASB). (2010). NIC 32. Instrumentos financieros: presentación. London UK: IASB.

International Accounting Standards Committee Foundation. (2009). Normas Internacionales de Información Financiera, NIIF. Pronunciamiento oficial. London, UK: IASCF.

International Financial Reporting Standards. (s.f.). Members of the IASB. Recuperado de http://www.ifrs.org/The-organisation/ Members-ofthe-IASB/Pages/Members-of-the-IASB Aspx

Limas, S. J. \& Higuera, J. A. (2012). Impacto socioeconómico del sector de la economía solidaria en el departamento de Boyacá, Colombia 2000- 2009. Apuntes del CENES, 111-140. 
López, C. E. \& Ochoa, H. (2002, sep.-dic.). Políticas subnacionales de fomento a la economía social en Venezuela. Revista de Ciencias Sociales (RCS), 8(3).

López, M. C. (2005). La influencia del valor razonable de los bienes inmuebles en el análisis de los estados financieros de la empresa inmobiliaria. Tesis doctoral. Departamento de Economía Financiera y Contabilidad, Facultad de Ciencias Económicas y Empresariales Universidad de Granada.

Mantilla, S. (2011). Estándares. Normas Internacionales de Información Financiera IFRS/NIIF. Bogotá: Ecoe.

Martínez, M. D. C. B., Pascual, M. E. V. \& Gallego, A. G. (2012). El impacto del uso del valor razonable sobre las empresas. Revista contable, (5), 74-85.

Mateo, J. (1985). Historia de la reforma de los principios cooperativos. Revesco, Estudios Cooperativos, 37-68.

Monzón, J. L. \& Defourny, J. (s.f.). La economía social, entre economía capitalista y economía pública. Recuperado de www.cfg.uchile.cl

NICNIIF (2011, 13 de mayo). El IASB y FASB publican una guía sobre la medición a valor razonable y los requisitos de revelación. Recuperado de http://www.nicniif.org/home/novedades/el-iasb-y-fasb-publicanuna-guia-sobre-medicion-al-valor-razonable-y-los-requisitos-de-revelacion.html 
Orejuela, F. \& Portocarrero, J. (2012). La economía solidaria en Colombia: obstáculos normativos y su enfoque económico. Magazín Empresarial, 23-36.

Paniagua, M. (2006). El capital social cooperativo en derecho español y su armonización con las normas internacionales de contabilidad. Revesco (90), 57-91.

Polo, F. (2006). La contabilidad de cooperativas en un proceso de armonización contable internacional. El caso de España. Revesco (89), 108-138.

Polo, F. (2007, agosto). Impactos de las normas internacionales de información financiera en el régimen económico de las sociedades cooperativas. CIRIEC-España, Revista de Economía Pública, Social y Cooperativa, (58), 83-108.

Rendón, Rodríguez \& Riascos, P.A. (2013). Análisis del impacto en el capital institucional de las cooperativas de ahorro y crédito frente a la aplicación de las NIIF. Caso cooperativas del Valle del Cauca. Cuadernos de Contabilidad, 14 (36) 881-901.

Salazar-Baquero, E. (2011). Análisis de las implicaciones no financieras de la aplicación de la NIIF para pymes en las medianas entidades en Colombia. Cuadernos de Contabilidad, 12, 211-241.

Sánchez, S. (2003). Plan contable para las sociedades cooperativas: comentarios y consideraciones. Técnica Contable (654), 13-21. 
Slof, E.J., Rodríguez, G., Torvent, M., Villadell, I. \& Sola, M. (2005, 22 a 24 de septiembre). Armonización y gobierno de la diversidad. En XIII Congreso AECA, Oviedo.

Superintendencia de la Economía Solidaria. (2008). Circular básica jurídica. Recuperado de http://www.supersolidaria.gov.co/es/normativa/circular-basica-juridica

Superintendencia de la Economía Solidaria. (s.f.). Entidades vigiladas que reportan información. Recuperado de http://www.supersolidaria.gov. co/es/entidad/entidades-vigiladas- que-reportan-información

Zubiaurre, M. A. (2004). Sociedades cooperativas. Aspectos contables singulares. Cuadernos de Gestión, 4 (2), 47-62.

Zúñiga, F., Pacheco, L. \& Díaz, J. (2009). Convergencia contable: cambios profundos en la contabilidad chilena. Activo fijo, un caso a considerar. Capic Review, 7, 75. 